\title{
Exploring the Involvement of FoxM1 in CML Cells from DNA Damage Response-Regulatory Prospective
}

Lin Du ( $\nabla$ dlxyph@163.com )

Xingyi People's Hospital https://orcid.org/0000-0002-8572-4360

Manli Wang

Xingyi People's Hospital

Hui Li

Chengdu First People's Hospital

\section{Fang Wang}

The First Affiliated Hospital of Xi'an Jiaotong University

\section{Research Article}

Keywords: Chronic myeloid leukemia, Bcr/Abl fusion protein, DNA damage response, FoxM1

Posted Date: April 20th, 2021

DOl: https://doi.org/10.21203/rs.3.rs-411734/v1

License: @ (i) This work is licensed under a Creative Commons Attribution 4.0 International License. Read Full License 


\section{Abstract \\ Background}

FoxM1 is widely accepted as an oncogenesis factor, for it is one of the most frequently upregulated genes in a broad spectrum of human malignancies. Herein, we presented the status of FoxM1 in CML samples and cell lines.

\section{Methods}

We compared FoxM1 abundance and phosphorylation using PB-MNC samples from CML patients and healthy donors. DNA damage response (DDR) was investigated in the presence of oncogene or chemical. Through enforced expression of FoxM1 or lentivirus mediated silencing, we explored the participation of FoxM1 in DDR regulation.

\section{Results}

Overexpression of FoxM1 was only observed in 3 samples from patients of advanced stage. However, hyper-phosphorylation of FoxM1 was evidently detected in the CML cohort. Furtherly, the DNA damage response that in accompany with the formation of $\mathrm{Bcr} / \mathrm{Abl}$ was responsible for the rise in FoxM1 phosphorylation. Bcr/Abl provoked a modest extent of DNA damage, which, in turn, roused the repair system, mirrored by phosphorylation of the ATM/ATR-CHK1/2 axis. FoxM1 was a downstream target of

CHK1 which directly associated with FoxM1 in the presence of DNA damage. Activation of FoxM1 served as a DDR regulator by inducing the expression of Rad51 and Brca1, genes that participated in DNA repair. Depletion of FoxM1 impaired DNA repair, leading to cell cycle arrest in G2/M phase and the onset of apoptosis in a P53-dependent fashion. Finally, our data demonstrated that phosphorylation of FoxM1 did not rely on Bcr/Abl kinase activity. Suppression of FoxM1 showed lethal potential to primary CML cells, and most importantly, the lethality was not affected by the TKls insensitiveness.

\section{Conclusions}

Abnormality in FoxM1 activity in CML cells enlightened us that constantly present DNA damage rendered leukemia cells more reliant upon the DNA damage repair system. Targeting FoxM1 could be exploit as an alternative strategy to overcome TKIs resistance.

\section{Introduction}

Chronic myeloid leukemia (CML) is a clonal myeloproliferative disease, originated from hematopoietic stem cells (HSCs). Bcr/Abl fusion protein encoded by the Philadelphia chromosome ( $\mathrm{Ph}$ ) is the unique hallmarks of CML cells. As a non-receptor tyrosine kinase, the fusion protein with constitutive activity 
activates a series of downstream signals, contributing to uncontrolled proliferation, growth factorindependent survival, modified cell adhesion, and apoptosis inhibition. Clinically CML progresses through three distinct phases: a relatively benign chronic phase (CP) representing $85-90 \%$ of patients at diagnosis, followed by an accelerated phase (AP), and finally to either a myeloid or lymphoid blast crisis (BC). Upon the innovation of tyrosine kinase inhibitors (TKIs) which selectively inhibits Bcr/Abl kinase, $\mathrm{CML}$ becomes the best example of successful targeted therapy. However, emerging IM resistance and therapeutic failure is present in more than one fourth of patients with CP-CML, and is more commonly seen $\mathrm{BP}-\mathrm{CML}^{[1-3]}$.

The mammalian transcription factor (TF) Forkhead Box M1 (FOXM1) is one of the members of the Forkhead superfamily. Typically, FoxM1 is ultimately associated with proliferation, and is ubiquitously expressed in proliferating tissues. FoxM1 facilitates cell cycle progression by promoting the S-phase entry and G2/M transition, as well as proper execution of mitosis. As a proliferation-specific transcriptional factor, FoxM1 stimulates expression of a variety of genes. For instance, transcriptional activation of cyclin B by FoxM1 is critical for timely mitotic entry, and CENP-F, another target of FoxM1, is required for precise functioning of the mitotic spindle checkpoint ${ }^{[4]}$. In addition, Plk1, Cyclin B1, and Aurora B, all of which required for mitotic progression are transcriptional regulated by FoxM1 ${ }^{[5]}$. Given this property, it is quite reasonable that aberration in FoxM1, transcription or activity, is one of the most common events in a broad range of human cancers ${ }^{[6,7]}$. In general, high FOXM1 abundance correlates with tumor progression and aggressiveness. Along with promoting cell proliferation, FoxM1 can protect cells from genotoxic agent-induced senescence by enhancing DNA repair capacity. Extra DNA breaks is often observed in cancer cells, burdening them with cytotoxicity executed by DDR system. FoxM1, upregulated under such circumstance, shifts the signals from cycle arrest and apoptosis induction to survival by facilitating DNA damage repair.

In the present study, we focused on the pro-survival contribution of FoxM1 from the DDR-regulatory perspective in CML clinical samples and cell lines. Our data suggested that DDR signals were constitutively activated in CML cells due to persistence of DNA damage in company with the formation of $\mathrm{Bcr} / \mathrm{Abl}$ fusion gene. FoxM1 played vital role in maintaining cell viability by stimulating Rad51 and Brca2 gene expression to promote damage repair. Depletion FoxM1 provoked cell death in both TKIs sensitive and insensitive CML cells. Conclusively, our study pointed out that DDR signals could be weaponized to kill CML cells by interfering FoxM1.

\section{Materials And Methods}

2.1 Regents. Reagents including antibodies, kits, chemicals (with work concentration) were all stated in suppl. table 2 .

2.2 Cell culture. K562, Ba/F3-B/A and Ba/F3 cells were cultured in RPMI-1604 medium (HyClone, South Logan, UT, USA) supplemented with $10 \%$ of FBS (Biological Industries, Kibbutz Beit Haemek, Israel) (V/V). For Ba/F3 cells, IL-3 (PeproTech, Rocky Hill, NJ, USA) at 70 ng/ml was added. HEK-293 cells were cultured 
in DMEM medium (HyClone, South Logan, UT, USA) supplemented with $10 \%$ of FBS (Biological Industries, Kibbutz Beit Haemek, Israel) (V/V). All cells were maintained in $37{ }^{\circ} \mathrm{C}, 5 \% \mathrm{CO}_{2}$.

2.3 Isolation of PB-MNCs. Peripheral blood mononuclear cells (PB-MNCs) were isolated using human bone marrow mononuclear cell isolation kit (Tbd science, Tianjin, China) according to users` guide. Sample information was listed in Table S1. Informed consent forms were signed by all patients and healthy donors to allow the use of their cells for experiments and the experiments were performed in accordance with the regulation of institutional ethics committee of the First affiliated hospital of Xi'an Jiao tong university. Isolated cells were seeded in StemProTM-34 SFM (Gibco, Grand island, NY, USA), and used for following experiments immediately ${ }^{[8]}$.

2.4 CCK-8 assay. Cells were seeded in 96-well plate (in the abovementioned mediums), etoposide ( $8 \mu \mathrm{M})$ was then added to make the final volume to $200 \mu \mathrm{L}$, for the duration of indicated days, $20 \mu \mathrm{L}$ of enhanced CCK-8 was added, and the plate was then incubated in $37^{\circ} \mathrm{C}$ for half hour. OD450 was then measured using microplate readers (BioTek, Biotek Winooski, Vermont, USA).

2.5 FoxM1 knockdown. hFOXM1 shRNA lentiviral was a product of Santa Cruz Biotechnology (Santa Cruz, CA, USA). Cells were plated in 6-well plate $\left(5 \cdot 10^{5} / \mathrm{ml}\right)$ in complete medium with $5 \mu \mathrm{g} / \mathrm{ml}$ of polybrene (Santa Cruz, CA, USA), after 30 min of incubation in $37^{\circ} \mathrm{C}, 4 \mu \mathrm{L}$ of the stock solution of the FoxM1 shRNA lentiviral or scrambled control was added. The plate was then centrifuged at $150 \cdot \mathrm{g}$ at room temperature for 4 hours, followed by 48 hours incubation in $37{ }^{\circ} \mathrm{C}, 5 \% \mathrm{CO}_{2}$. cells were collected for cycle and apoptosis analysis or protein extraction.

2.6 Construction of FoxM1 plasmid. Full-length FoxM1 was cloned from heathy white blood cells, double digested with EcoR】 and Xho囚 TaKaRa (Tokyo, Japan), and cloned into pCMV-N-Flag backbone vector using T4 ligase TaKaRa (Tokyo, Japan). The ligation product was then transformed into competent DH5a cells and selected by colony PCR and double digestion, followed by sequencing.

2.7 Construction of CHK1 plasmid. Wide type CHK1 plasmid was constructed as FoxM1. The mutants were built by overlay PCR using the wide type as template. The primers were listed in suppl. materials. After obtaining the mutant DNAs, the followed steps (double digestion, ligation, transforming and selection) were as described in 2.6 section. Transfection was conducted using Lipofectamine ${ }^{\circledR} 3000$ (Invitrogen, Carlsbad, CA, USA) according users' manual. For each well of 6-well plate containing $5 \cdot 10^{5} / \mathrm{ml}$ cell, $2 \mathrm{ng}$ of plasmid was used.

2.8 Western blotting. Cells post indicated treatments were harvested, washed three times with pre-cold PBS, lysed in RIPA buffer supplemented with protease and phosphatase inhibitor cocktail (Beyotime, Hangzhou, China. 1:50 v/v) on ice for 15 minutes, centrifuged at $12000 \mathrm{rpm}$ at $4^{\circ} \mathrm{C}$ for 5 minutes. The supernatants were collected for protein quantification by BCA (Beyotime, Hangzhou, China). SDS-PAGE was performed to separate the protein, briefly, $50 \mathrm{ng}$ of protein for each well was loaded, electrophoresis using $10 \%$ of gel was ran for 2 hours at $120 \mathrm{~V}$. The proteins were then transferred to PVDF membrane at 
constant current of $250 \mathrm{~mA}$. Then the membrane was blocked in $5 \%$ of non-fat milk for 1 hour in room temperature and incubated with indicating antibodies (see suppl. table 2) at $4{ }^{\circ} \mathrm{C}$ overnight, washed in TBST buffer for three times, followed by secondary antibody incubation (1 hour, RT). The signals were detected using Odyssey ${ }^{\circledR}$ CLx Imaging System (LI-COR biosciences, Nebraska, USA).

2.9 Co-Immunoprecipitation. Co-Immunoprecipitation was performed according to the users' guide. Briefly, cells were lysed, and the lysate was pre-cleaned using control agarose resin followed by incubation with $\mathrm{CHK} 1$ or Flag antibodies to form immune complex which was then captured by protein A/G plus agarose, and centrifuged to separate the uncombined FoxM1. The pellets were washed and resuspended with non-reducing lane marker sample buffer, boiled and centrifuged to collect elution for western blotting analysis.

2.10 Statistical analysis. The data were presented as $X \pm S D(n=3)$. The difference between each group was evaluated using Student's t-test. $P<0.05$ was considered statistically significant.

\section{Results}

\subsection{Phosphorylation of FoxM1 in the presence of Bcr/Abl fusion protein.}

Previous studies have shown that FoxM1 overexpression was not only involved in various malignancies, including cancers of the liver, prostate, brain, breast, lung, colon, pancreas, cervix, ovary, but it was also proposed to be an early hallmark during carcinomagenesis ${ }^{[6]}$. We herein, detected the mRNA and protein levels of FoxM1 in CML-patient derived peripheral blood mononuclear cell (PB-MNCs). In comparison with heathy counterparts, PB-MNCs from CML did not show evident elevation at both mRNA and protein levels (Fig. 1. AB). However, our data revealed relatively higher level of FoxM1 in 3 individuals that either at blast crisis or with recurrent disease (Fig. 1. AB, in circle. Suppl. table 1). Considering the possibility that the activity of FoxM1 may regulated by post-translational modifications, we next examined the phosphorylation of FoxM1 using these samples, we found that the clearly enhanced phosphorylation in $\mathrm{CML}$ samples (Fig. 1. C). These results indicated that formation of $\mathrm{Bcr} / \mathrm{Abl}$ oncogene was more likely to alter the modification of FoxM1. We further investigated this finding in a cell line model, mouse pro-B cell, $\mathrm{Ba} / \mathrm{F} 3$. With expression of bcr/abl, Ba/F3-B/A cells showed no sign of increased FoxM1 induction, but the phosphorylation was obviously augmented (Fig. 1. D).

\subsection{Bcr/Abl stimulated FoxM1 phosphorylation through DNA damage response.}

FoxM1 has been documented as a master regulator of DNA damage response, and activation of oncogene may trigger DNA damage ${ }^{[9]}$. We thus tested the activation of DDR in patients' samples and found that the abundance of $\mathrm{Y}-\mathrm{H} 2 \mathrm{AX}$ was significantly increased (Fig. 2. A). This result was subsequently confirmed in cell line (Fig. 2B), in which bcr/abl was the unique variable to rule out the possibility that 
boosted activity of FoxM1 was resulted from alternative genetic abnormities. We also found that the ATR/CHK1 and ATM/CHK2, two critical signaling axes that governed the DDR, were activated in Ba/F3$\mathrm{B} / \mathrm{A}$ cells (Fig. $2 \mathrm{C}$ ). These results were indicative of involvement of DDR in cellular transformation induced by Bcr/Abl. We next focused on the molecular connection between the DDR onset and phosphorylation of FoxM1. To construct the DDR model, we treated Ba/F3 cells with etoposide based on the observations that etoposide induced apoptosis by provoking DNA damage. However, the concentration of etoposide we used was insufficient to trigger cell death but enough to engage DDR. Hopefully, etoposide of $8 \mu \mathrm{M}$ (Fig. 2. D upper) would mimic the pro-DDR effect without cell death in cells harboring oncogene. Key regulators and DNA damage marker were analyzed in the etoposide scenario to confirm the commencing of DDR, both CHK1 and CHK2 pathways were involved and raise of FoxM1 amount and phosphorylation were detected (Fig. 2E). However, we could not ignore the fact that with even relatively low-dose of etoposide cell growth was remarkably inhibited (Fig. 2. D lower), which was conflicting with characters of enhanced proliferation in oncogene-transformed cells. This contradiction could be interpreted by nature of oncogene, by which the DDR severed as an adaptive strategy to prevent the DNA damage from reaching the lethal extent of genomic instability. Meanwhile, the main consequence of the Bcr/Abl was activation of multiple signaling that in favor of cell survival and proliferation. This was essentially different from etoposide that relied on the DDR to restrict cell growth and/or achieve apoptosis. Nevertheless, application of the etoposide revealed that FoxM1 activation was associated with DDR.

\subsection{FoxM1 was activated by direct association with CHK1}

Based on the abovementioned findings, we further asked which molecule among the DDR cascade controlled FoxM1 activity. We first used inhibitors that targeted five key participants of DDR to block its activation in the presence of $\mathrm{Bcr} / \mathrm{Abl}$. CHK2 was previously reported to mediate FoxM1 phosphorylation and stabilization ${ }^{[10]}$, while in our study, FoxM1 phosphorylation was diminished maximally when the CHK1 inhibitor was administrated, suggesting the regulating role of CHK1 on FoxM1(Fig. 3. A). Meanwhile, FoxM1 phosphorylation was also weakened by ATR inhibition. We supposed that the ATR contribution to FoxM1 activity may require $\mathrm{CHK} 1$ that acts as a downstream substrate ${ }^{[11]}$. Suppression of ATR leaded to dephosphorylation of FoxM1 was a consequence of CHK1 inactivation. Next, we observed direct interaction of FoxM1 and CHK1 in Ba/F3-B/A cells (Fig. 3. B). Further, under a chemical-induced DDR setting, this interaction was present in Ba/F3 cells (Fig. 3. C), and sharply attenuated when the CHK1 inhibitor was introduced (Fig. 3. D). Combined with the result in Fig. 3.A, we concluded that CHK1 promoted FoxM1 activity through direct association. As a kinase in the DDR process, function of the CHK1 relied on the phosphorylation on multiple sites ${ }^{[12]}$. In this study, we compared 3 modification sites on CHK1 under oncogenic and chemical-induced DDR condition. all the three sites were affected by both by oncogene and chemical (Fig. 3. E). To explore the exact site(s) that involved in FoxM1 activity, 4 phosphorylation-dead mutants of CHK1 were constructed and expressed in HEK293 cells followed by etoposide administration to induce DDR, under which the mutants were forced to associated with FoxM1. S269A mutant fully abrogated the association, suggesting its vital role in FoxM1 activation (Fig. 3.F). 


\subsection{FoxM1 protected cells by rapid DNA damage repair.}

The DDR has been harnessed to trigger cell death in the discovery of many anti-cancer strategies. Pause in cell cycle during this process is intrinsically a cell-protective response, allowing cells to fix the damage induced by chemicals or radiation assaults. In this regard, we asked how cells deal with the reaction without decrease in proliferation rate (Fig. 4. I). Firstly, we found that FoxM1 depletion abate cell growth (Fig. 4. A), in day 3, cell viability was below the input level, indicating the initiation of cell death. The FCM results then revealed that without FoxM1, cell cycle was arrested in G2/M phase (Fig. 4. B). Apoptosis was also detected and amplified along time (Fig. 4. C). Modulation of DNA repair by FoxM1 was investigated next, amount of Rad51 and Brca2, two major DNA repair executors, were evidently reduced in $\mathrm{Ba} / \mathrm{F} 3-\mathrm{B} / \mathrm{A}$ cells (Fig. 4. D), indicating that FoxM1 may play as transcription factor that promotes expression of Rad51 and Brca2 during oncogene-driven DDR. Moreover, recovery from DNA damage was further studied under the chemical condition. Cells treated with etoposide $(8 \mu \mathrm{M})$ for 12 hours to provoke DDR, and the drug was then withdrew followed by detection of $\mathrm{Y}-\mathrm{H} 2 \mathrm{AX}$ levels. Without FoxM1, it took more than 9 hours for $\mathrm{Y}-\mathrm{H} 2 \mathrm{AX}$ to reach the basal level, in comparison, the duration of $\mathrm{y}-\mathrm{H} 2 \mathrm{AX}$ was less than 6 hours with FoxM1 (Fig. 4. E). Additionally, FoxM1 was further enforcedly expressed in K562 cells (Fig. 4.F), in which, Rad51 and Brca2 were up-regulated with etoposide (Fig. 4. G), further suggesting positive role of FoxM1 in Rad51 and Brca2 upon DDR. We finally analyzed the cell cycle profile under the circumstance of enforced expression of FoxM1, which not only accelerated G2/M transition, but restored cell from G2/M arrest also post etoposide release (Fig. 4. H). Taken together, our data clearly demonstrated a cytoprotective role of FoxM1, which played as a transcription factor to induce Rad51 and Brca2 expression, resulting to boosted DNA repair that facilitate cell avoiding apoptosis or growth arrest under DNA damage induced by either oncogene or chemicals.

\subsection{Repression of FoxM1 induced apoptosis in imatinib- insensitive CML cells.}

Since the hyper-phosphorylation of FoxM1 in Bcr/Abl-expressing cells, survival or apoptotic advantages that attributed to this feature was our final concern. We first asked if the modification was Bcr/Abl kinase dependent. Samples from $6 \mathrm{CML}$ patients, containing 3 that resistant to imatinib treatment (No. 25,27,29. See in suppl. table 1), were used for the investigation. The data clearly showed no sign of descent in pFoxM1 levels in all 6 samples after imatinib treatment ( $1 \mu \mathrm{M}, 24$ hours), indicating that FoxM1 phosphorylation was not addicted to Bcr/Abl, at least, the kinase activity (Fig. 5.A). To analyze the proapoptotic effect of FoxM1 suppression, FoxM1 inhibitor, RCM-1 ${ }^{[13]}$ was firstly used. Consistent with the finding in cell line (Fig. 4.C), cells from all 6 patients, regardless of the imatinib sensitiveness, responded to FoxM1 blockage (Fig. 5.B). FoxM1 silence were then applied in 3 imatinib-resistant cells to further confirm this observation (Fig. 5.C). Connection between FoxM1 and DNA repairing capacity was measured next. Inhibition of Bcr/Abl did not alter the $\mathrm{Y}$-H2AX levels, which, however, dramatically ascended in the absence of FoxM1 (Fig. 5.D). Similar to that found in cell line, FoxM1 knockdown suppressed the expression of Rad51 and Brca2, but caused more robust DDR, reflected by enhanced phosphorylation of ATM/ATR/CHK1/CHK2 (Fig. 5.E). We also found that without FoxM1, phosphorylation 
on Ser46 of P53, an apoptosis-decisive modification ${ }^{[14]}$, was sharply augmented, furthermore, the nuclear translocation of P53 was detected. Finally, P53 targeted genes, Puma and Noxa, were upregulated, cleaved caspase-3 and Parp were also observed (Fig. 5.F). These results suggested that the cell death was P53 dependent, at least, partly.

\section{Discussion}

The DDR pathway is evolutionarily developed by cells for dealing with a variety of exogenous and endogenous insults that lead to DNA breaks. In nature, the purpose of this processes is to maintaining genomic stability by either fixing the damage or triggering programmed cell death to remove cells harboring irreparable damage. In this respect, DDR serves as an anti-cancer barrier in early human tumorigenesis. The consequence of DDR activation is most likely dependent on the extent of DNA damage $\mathrm{e}^{[15-17]}$. Thus, cytotoxic level of damage has been long applied in the innovation of strategies for treating and preventing cancer. On the one hand, traditional approaches, such as radiotherapy and platinum drugs, are based on direct generation of irreparable damage. Accordingly, abnormality in repairing system is most frequently seen in refractory malignancies. On the other hand, piloting the DDR towards apoptosis susceptible by interfering critical modulators has ushered in a new era of research. Inhibitors that targeting ATM, ATR, CHK1 and CHK2 mirror the expansion of therapeutic landscape ${ }^{[18]}$.

Up-regulation of FoxM1 is frequently accompanied with uncontrolled growth and altered susceptibility to apoptosis $^{[19,20]}$, key characteristics in human malignancies. Therefore, FoxM1 has been widely accepted as a potent oncogenic factor that is essential for cancer development and progression. FoxM1 acts functionally as a transcription factor that drives the expression of a wide spectrum of genes required in cell proliferation and cell cycle regulation. Evidences also suggest that FomX1 is an emerging master regulator of DNA damage response. This function is reflected by the ability of FOXM1 to transcriptional controlling of a network of damage repairing genes ${ }^{[21,22]}$.

TKIs resistance has been the major obstruction for curing CML, studies focused on overcoming this issue is in urgent need. We herein report FoxM1 as a potent target that may ameliorate the management of CML. We explored the biology of FoxM1 in CML cells and its involvement in cell biological features. Unlike previous findings, upregulation of FoxM1 was only observed in 3 patients at advanced stage, implying the indicative role of FoxM1 expression in disease progression. Recent study revealed that the FoxM1 expression could be stimulated by ROS generated during oncogenesis ${ }^{[23]}$, meanwhile, the $\mathrm{PI3K} / \mathrm{mTOR}$ pathway downstream Bcr/Abl is a driving force for ROS production ${ }^{[24]}$. As a mediator of DDR, FoxM1 expression is also strengthened by DNA damage ${ }^{[25]}$, which aroused in Bcr/Abl-expressing cells in a number of ways ${ }^{[26,27]}$. All these works are in favor of upregulation of FoxM1 in CML cells. This conflict may be owing to the transcriptional dynamic of FoxM1 which exclusively expressed in proliferating cells, and the CML-CP is characterized by terminally differentiated cells in which FoxM1 expression is excluded. 
Fine tuning of FoxM1 function is closely interrelated with layers of post-translational modifications. Transcriptional activity of FoxM1 is tightly regulated during cell division by multisite phosphorylation [28] Phosphorylation status of FomX1 is in constant fluctuation along with the progress of cell cycle, from relative hypo-phosphorylation in G1/S phase to hyper-phosphorylation in M phase. Various kinases and its counteracting phosphatases are participated in the dynamic of FoxM1. For example, G1/S transition is closely attached with phosphorylation on multiple sites, including T620, T627, and S672 by Cyclin DCDK4/6 complexes ${ }^{[29]}$. In addition, transactivation activity of FoxM1 relies heavily on T611 phosphorylation, which occurred in $\mathrm{G} 2$ phase by cyclin A/E-CDK2 complexes ${ }^{[30]}$. In response to DNA damage, CHK2 phosphorylates FoxM1 at S361, maintaining its stability and increasing the expression of Brca2 genes ${ }^{[10]}$. These studies support that abnormality in FoxM1 activity is more than a mere consequence of high proliferation rates, and that also actively participates as a cytoprotective factor under stress condition. We demonstrated herein that the S361 site phosphorylation was more likely attributed to $\mathrm{CHK} 1$ by direct interaction. CHK1 is also an important DDR regulator, and causes cell cycle delay by governing CDK activity through cdc25 phosphatase ${ }^{[31]}$. Recent study confirmed its overexpression in CML and highlighted the potential in overcoming TKIs resistance ${ }^{[32]}$. There is also evidence showing that enhanced $\mathrm{CHK} 1$ activity in $\mathrm{CML}$ cells protects cells from genotoxic agents ${ }^{[33]}$. Our data suggest that FoxM1 is a downstream target of CHK1, which, therefore, resides at the crossroad of DNA repair and cell cycle checkpoint under the pressure of DNA damage. FoxM1 activation may result from a joint effect of both $\mathrm{CHK} 1$ and $\mathrm{CHK} 2$, since inhibition of CHK2 also dephosphorylated FoxM1. Thus, harnessing DDR through suppressing FoxM1 seems to be a better option than targeting CHK1, for it eliminate the possibility that CHK2 may ascent as major path to compensate the loss the CHK1.

Taken together, these findings explicitly prompt that modest DNA damage produced as by-effect of oncogenesis is compatible with cancer cells, in which, repair capacity holds the key to cell fate decision. Relatively more robust DDR system counteracts with the cytotoxic effects of DNA damage. Meanwhile, the damage that frequently observed in cancer cells offers the source for additional mutation, which through the filter of selection pressures, renders cells more invulnerable to exogenous assaults. Abrogation of the system breaks the equilibrium between detriment of DNA damage and its remedy. As shown in Fig. 5. E two branches downstream the ATM/ATR kinase orchestrates the outcome of DNA damage induced by Bcr/Abl. FoxM1 accounts for remedying force, and unfortunately, dominates the whole network.

\section{Conclusions}

Our data suggest that constitutive phosphorylation of FoxM1 acts as an detoxifying factor to attenuate the oncogenic assaults to genome stability. FoxM1 suppression paves the path for manipulating DDR signals towards cell suicide, providing an alternative for overcoming imatinib resistance.

\section{Abbreviations}


DDR: DNA Damage Response

FoxM1: Forkhead Box Protein M1

CML: Chronic Myeloid Luekemia

ATM: Ataxia Telangiectasia Mutated

HSC: Hematopoietic Stem Cells

PB-MNC: Peripheral Blood Mononuclear Cell

ATR: Ataxia Telangiectasia And Rad3-Related Protein

CHK1: Checkpoint Kinase 1

CHK2: Checkpoint Kinase 2

TKIs:Tyrosine Kinase Inhibitors

Rad51: DNA Repair Protein RAD51 Homolog

Brca2: Breast Cancer Type 2 Susceptibility Protein

\section{Declarations}

Ethics approval and consent to participate: Informed consent forms were signed by all patients and healthy donors to allow the use of their cells for experiments and the experiments were performed in accordance with the regulation of institutional ethics committee of the First affiliated hospital of Xi'an Jiao tong university.

Consent for publication: Not applicable.

Availability of data and materials: The datasets generated during and/or analysed during the current study are available from the corresponding author on reasonable request.

\section{Competing interest: None}

Funding: The work was supported by the National Natural Science Foundation of Chain (No. 81600134), Basic Research Plan of Guizhou Heath Commission (gzwjkj2019-1-200), and State Key R\&D Plan of the Ministry of Science and Technology (2019YFF0216502).

Contributions: DL and WML designed the whole work, WML and LH performed the work, WF collected samples and performed the work. DL and W F wrote the manuscript. 


\section{References}

1. Cang S, Liu D: P-loop mutations and novel therapeutic approaches for imatinib failures in chronic myeloid leukemia. J Hematol Oncol 2008, 1:15.

2. HehImann R: How I treat CML blast crisis. Blood 2012, 120(4):737-747.

3. Meenakshi Sundaram DN, Jiang X, Brandwein JM, Valencia-Serna J, Remant KC, Uludag H: Current outlook on drug resistance in chronic myeloid leukemia (CML) and potential therapeutic options. Drug Discov Today 2019, 24(7):1355-1369.

4. Laoukili J, Kooistra MR, Bras A, Kauw J, Kerkhoven RM, Morrison A, Clevers H, Medema RH: FoxM1 is required for execution of the mitotic programme and chromosome stability. Nat Cell Biol 2005, 7(2):126-136.

5. Fu Z, Malureanu L, Huang J, Wang W, Li H, van Deursen JM, Tindall DJ, Chen J: Plk1-dependent phosphorylation of FoxM1 regulates a transcriptional programme required for mitotic progression. Nat Cell Biol 2008, 10(9):1076-1082.

6. Barger CJ, Branick C, Chee L, Karpf AR: Pan-Cancer Analyses Reveal Genomic Features of FOXM1 Overexpression in Cancer. Cancers (Basel) 2019, 11(2).

7. Gentles AJ, Newman AM, Liu CL, Bratman SV, Feng W, Kim D, Nair VS, Xu Y, Khuong A, Hoang CD et al: The prognostic landscape of genes and infiltrating immune cells across human cancers. Nat Med 2015, 21(8):938-945.

8. Wang F, Wang X, Li N, Liu J, Zhang L, Hui L, Feng A, Wang Z, Wang Y: Prolonged unfolded protein reaction is involved in the induction of chronic myeloid leukemia cell death upon oprozomib treatment. Cancer Sci 2021, 112(1):133-143.

9. Zona S, Bella L, Burton MJ, Nestal de Moraes G, Lam EW: FOXM1: an emerging master regulator of DNA damage response and genotoxic agent resistance. Biochim Biophys Acta 2014, 1839(11):13161322.

10. Tan Y, Raychaudhuri P, Costa RH: Chk2 mediates stabilization of the FoxM1 transcription factor to stimulate expression of DNA repair genes. Mol Cell Biol 2007, 27(3):1007-1016.

11. Zhao H, Piwnica-Worms H: ATR-mediated checkpoint pathways regulate phosphorylation and activation of human Chk1. Mol Cell Biol 2001, 21(13):4129-4139.

12. Goto $\mathrm{H}$, Kasahara K, Inagaki M: Novel insights into Chk1 regulation by phosphorylation. Cell Struct Funct 2015, 40(1):43-50.

13. Sun L, Ren X, Wang IC, Pradhan A, Zhang Y, Flood HM, Han B, Whitsett JA, Kalin TV, Kalinichenko VV: The FOXM1 inhibitor RCM-1 suppresses goblet cell metaplasia and prevents IL-13 and STAT6 signaling in allergen-exposed mice. Sci Signa/ 2017, 10(475).

14. Feng $L$, Hollstein $M, X u$ Y: Ser46 phosphorylation regulates p53-dependent apoptosis and replicative senescence. Cell Cycle 2006, 5(23):2812-2819.

15. Jackson SP, Bartek J: The DNA-damage response in human biology and disease. Nature 2009, 461(7267):1071-1078. 
16. Hanawalt PC: Historical perspective on the DNA damage response. DNA Repair (Amst) 2015, 36:2-7.

17. Pearl LH, Schierz AC, Ward SE, Al-Lazikani B, Pearl FM: Therapeutic opportunities within the DNA damage response. Nat Rev Cancer 2015, 15(3):166-180.

18. O'Connor MJ: Targeting the DNA Damage Response in Cancer. Mol Cell 2015, 60(4):547-560.

19. Joshi K, Banasavadi-Siddegowda Y, Mo X, Kim SH, Mao P, Kig C, Nardini D, Sobol RW, Chow LM, Kornblum HI et al: MELK-dependent FOXM1 phosphorylation is essential for proliferation of glioma stem cells. Stem Cells 2013, 31(6):1051-1063.

20. Chai N, Xie HH, Yin JP, Sa KD, Guo Y, Wang M, Liu J, Zhang XF, Zhang X, Yin H et al: FOXM1 promotes proliferation in human hepatocellular carcinoma cells by transcriptional activation of CCNB1. Biochem Biophys Res Commun 2018, 500(4):924-929.

21. Im J, Lawrence J, Seelig D, Nho RS: FoxM1-dependent RAD51 and BRCA2 signaling protects idiopathic pulmonary fibrosis fibroblasts from radiation-induced cell death. Cell Death Dis 2018, 9(6):584.

22. Khongkow P, Karunarathna U, Khongkow M, Gong C, Gomes AR, Yague E, Monteiro LJ, Kongsema M, Zona S, Man EP et al: FOXM1 targets NBS1 to regulate DNA damage-induced senescence and epirubicin resistance. Oncogene 2014, 33(32):4144-4155.

23. Park HJ, Carr JR, Wang Z, Nogueira V, Hay N, Tyner AL, Lau LF, Costa RH, Raychaudhuri P: FoxM1, a critical regulator of oxidative stress during oncogenesis. EMBO J 2009, 28(19):2908-2918.

24. Kim JH, Chu SC, Gramlich JL, Pride YB, Babendreier E, Chauhan D, Salgia R, Podar K, Griffin JD, Sattler M: Activation of the PI3K/mTOR pathway by BCR-ABL contributes to increased production of reactive oxygen species. Blood 2005, 105(4):1717-1723.

25. Nestal de Moraes G, Bella L, Zona S, Burton MJ, Lam EW: Insights into a Critical Role of the FOXO3aFOXM1 Axis in DNA Damage Response and Genotoxic Drug Resistance. Curr Drug Targets 2016, 17(2):164-177.

26. Skorski T: BCR/ABL, DNA damage and DNA repair: implications for new treatment concepts. Leuk Lymphoma 2008, 49(4):610-614.

27. Burke BA, Carroll M: BCR-ABL: a multi-faceted promoter of DNA mutation in chronic myelogeneous leukemia. Leukemia 2010, 24(6):1105-1112.

28. Liao GB, Li XZ, Zeng S, Liu C, Yang SM, Yang L, Hu CJ, Bai JY: Regulation of the master regulator FOXM1 in cancer. Cell Commun Signal 2018, 16(1):57.

29. Anders L, Ke N, Hydbring P, Choi YJ, Widlund HR, Chick JM, Zhai H, Vidal M, Gygi SP, Braun P et al: A systematic screen for CDK4/6 substrates links FOXM1 phosphorylation to senescence suppression in cancer cells. Cancer Cell 2011, 20(5):620-634.

30. Laoukili J, Alvarez M, Meijer LA, Stahl M, Mohammed S, Kleij L, Heck AJ, Medema RH: Activation of FoxM1 during $\mathbf{G} 2$ requires cyclin A/Cdk-dependent relief of autorepression by the FoxM1 $\mathrm{N}$-terminal domain. Mol Cell Biol 2008, 28(9):3076-3087. 
31. Neizer-Ashun F, Bhattacharya R: Reality CHK: Understanding the biology and clinical potential of CHK1. Cancer Lett 2021, 497:202-211.

32. Lei H, Jin J, Liu M, Li X, Luo H, Yang L, Xu H, Wu Y: Chk1 inhibitors overcome imatinib resistance in chronic myeloid leukemia cells. Leuk Res 2018, 64:17-23.

33. Nieborowska-Skorska M, Stoklosa T, Datta M, Czechowska A, Rink L, Slupianek A, Koptyra M, Seferynska I, Krszyna K, Blasiak J et al: ATR-Chk1 axis protects BCR/ABL leukemia cells from the lethal effect of DNA double-strand breaks. Cell Cycle 2006, 5(9):994-1000.

\section{Figures}

(A)

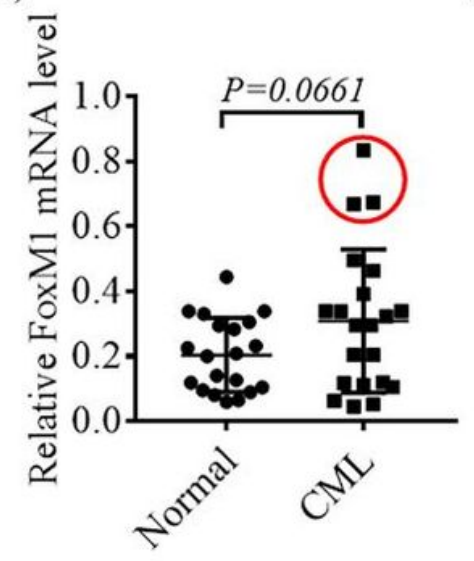

(B)

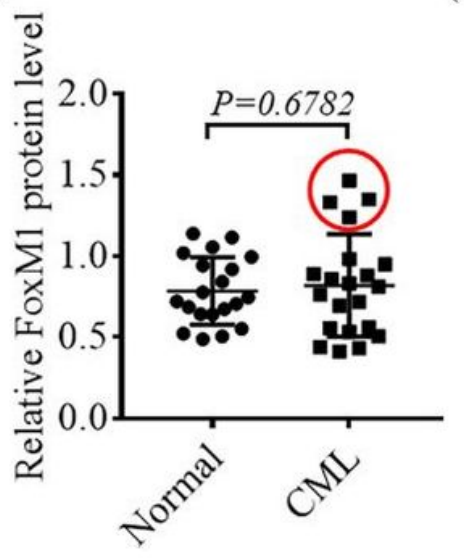

(C)

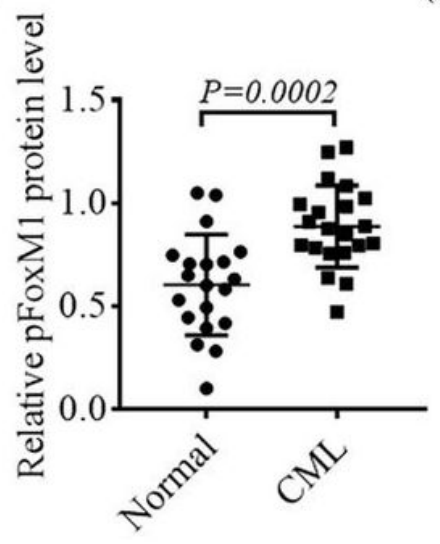

(D)

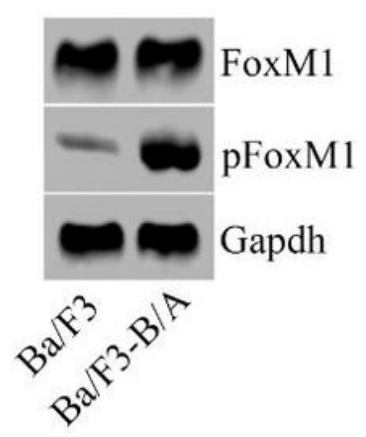

Figure 1

FoxM1 expression and phosphorylation. Comparison of FoxM1 mRNA (A), protein (B) and phosphorylation (C) in PB-BNCs from CML patients and healthy donors. Data were presented as FoxM1(p-FoxM1)/Gapdh ratio. (D) Bcr/abl fusion protein induced FoxM1 phosphorylation in cell line. 
(A)

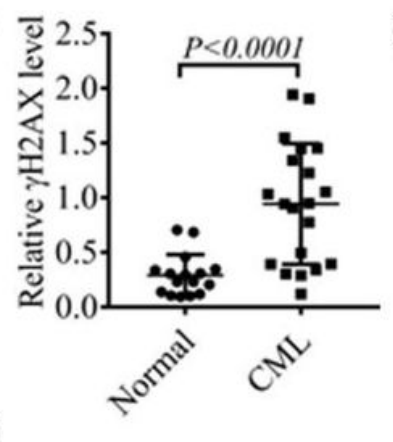

(B)

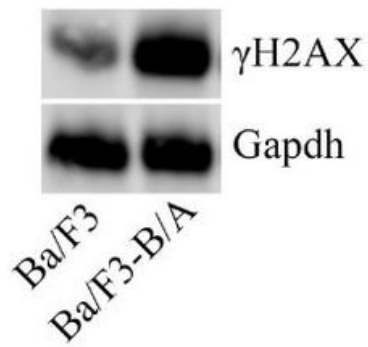

(C)

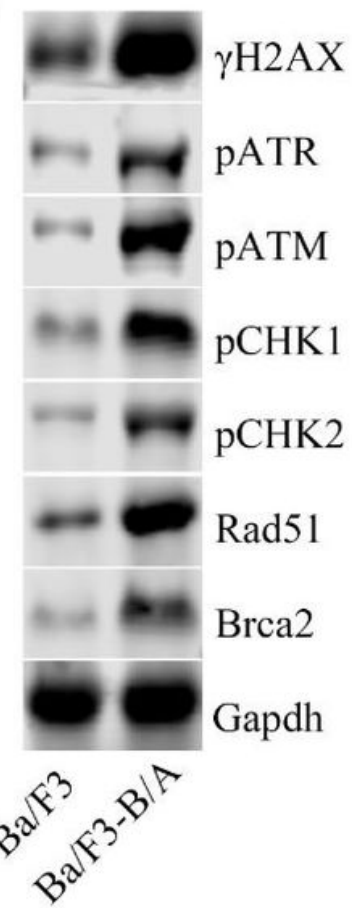

(D)
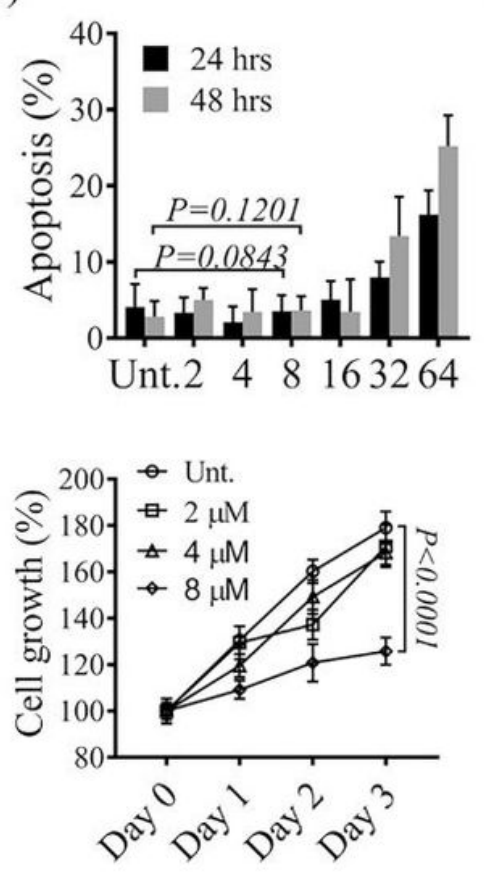

(E)
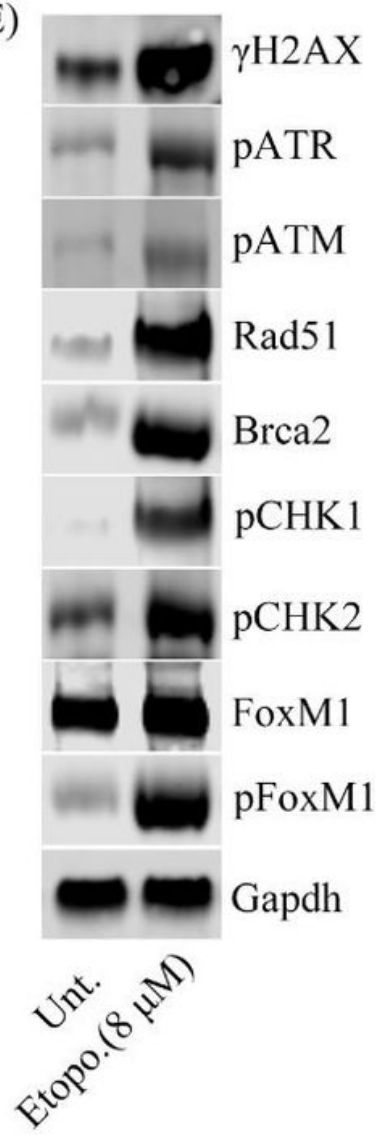

\section{Figure 2}

Bcr/Abl stimulated FoxM1 phosphorylation through DDR. (A). Induction of FoxM1 in the presence of $\mathrm{Bcr} / \mathrm{Abl}$ fusion protein. (B). Blockage of B/A did not alter the abundance of FoxM1. (C). DDR activation with formation of B/A. (D). Etopo. at $8 \mu \mathrm{M}$ failed to trigger apoptosis (Upper) but significantly inhibited cell growth (Lower). (E). Etopo. of $8 \mu \mathrm{M}$ induced DDR and FoxM1 upregualtion and phosphorylation. 
(A)

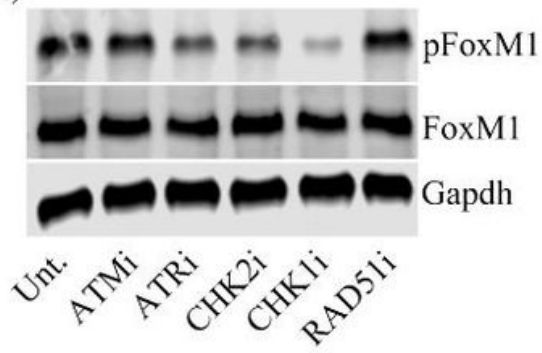

(F)

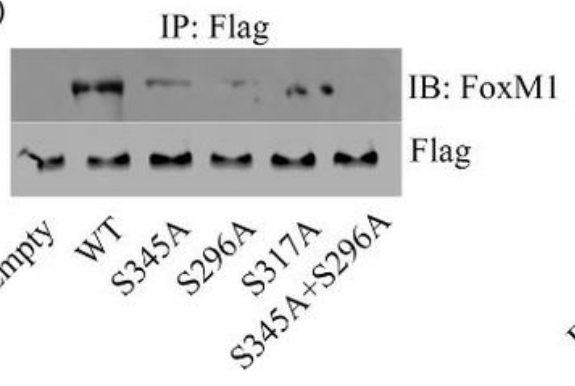

(B)

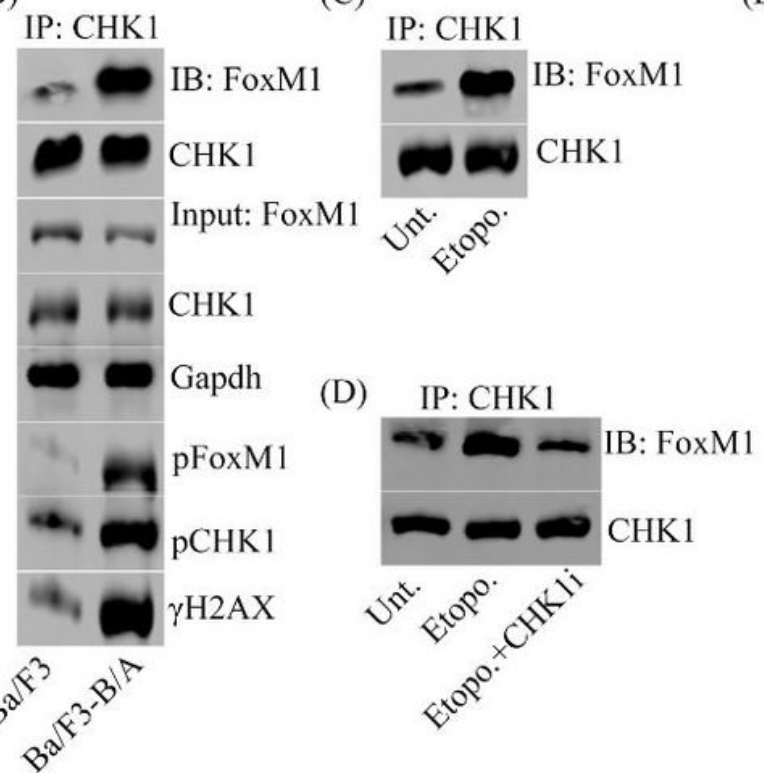

(E)

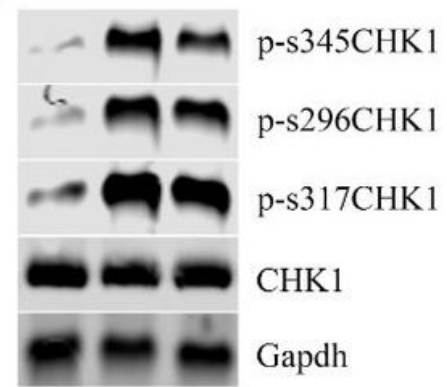

\section{Figure 3}

FoxM1 was activated by direct association with CHK1. (A). FoxM1 phosphorylation was inhibited by suppression of DDR (24 hours, working concentration of inhibitors were list in suppl. table 2). (B). Bcr/Abl promoted FoxM1 interaction with CHK1. (C) FomX1 interaction with CHK1 in Ba/F3 cells under DDR, which was then abrogated by CHK1 blockage (D). (E). Phosphorylated status of CHK1 in the presence of Bcr/Abl or etoposide (8 $\mu \mathrm{M}, 24$ hours). (F). Association of FoxM1 with CHK1 was dependent on phosphorylation of CHK1. 
(A)

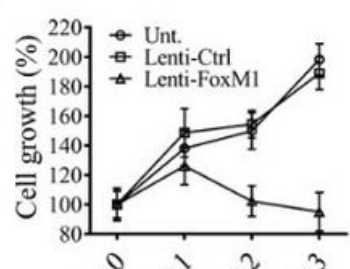

(B)

(C)
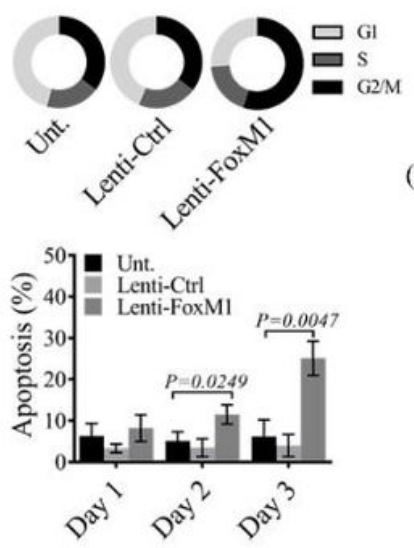

(D)

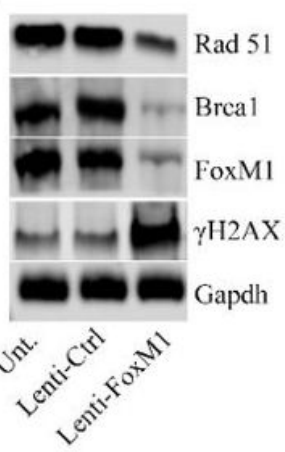

(E)

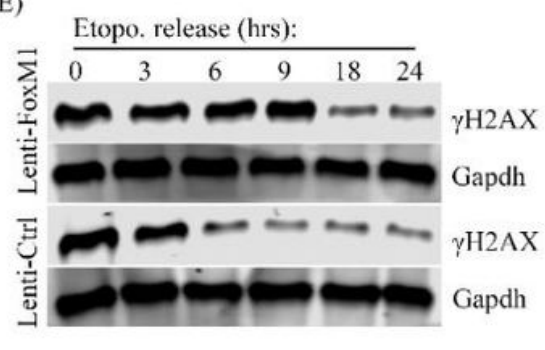

(F)

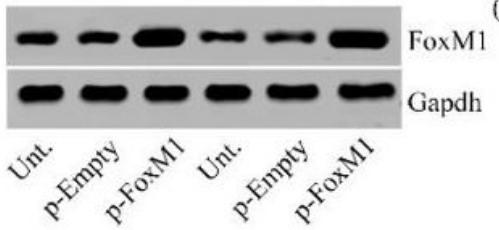

(G)

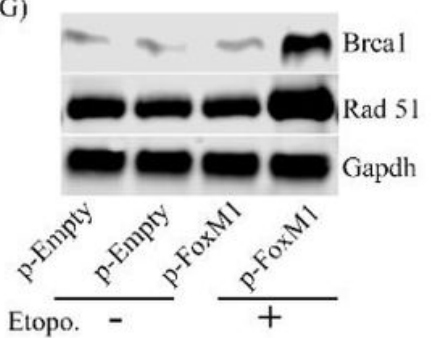

(H)

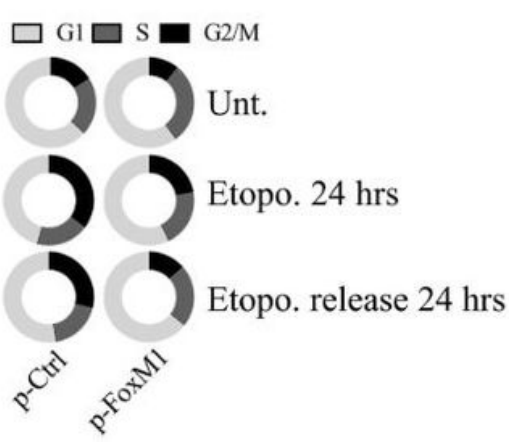

\section{Figure 4}

FoxM1 protected $\mathrm{CML}$ cell by rapid DNA damage repair. FoxM1 depletion resulted to Ba/F3-B/A cell growth inhibition (A), cell cycle arrest in $G 2 / M$ phase (B, 24 hours) and apoptosis (C, 48 hours). (D). FoxM1 depletion induced suppression of Rad 51 and BRAC expression in Ba/F3-B/A cells (48 hours). (E). FoxM1 depletion delayed DNA damage repair in Ba/F3-B/A cells (48 hours post lentivirus infection, cells were then treated with etoposide for another 12 hours and released for the indicating times). (F). Enforced expression of FoxM1 in K562 cells and upregulated Rad 51 and BRAC in the presence of etoposide (G, 48 hours). (H). Restored cell cycle from G2/M arrest by etoposide. 
(A)

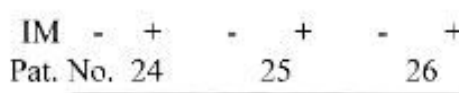

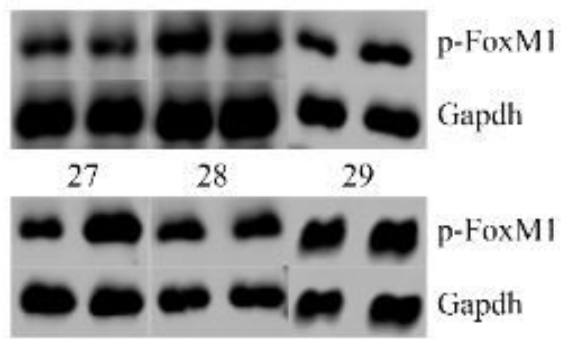

(B)

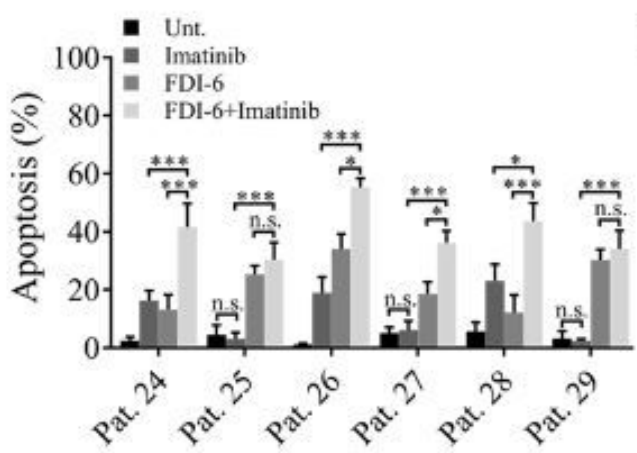

(F)
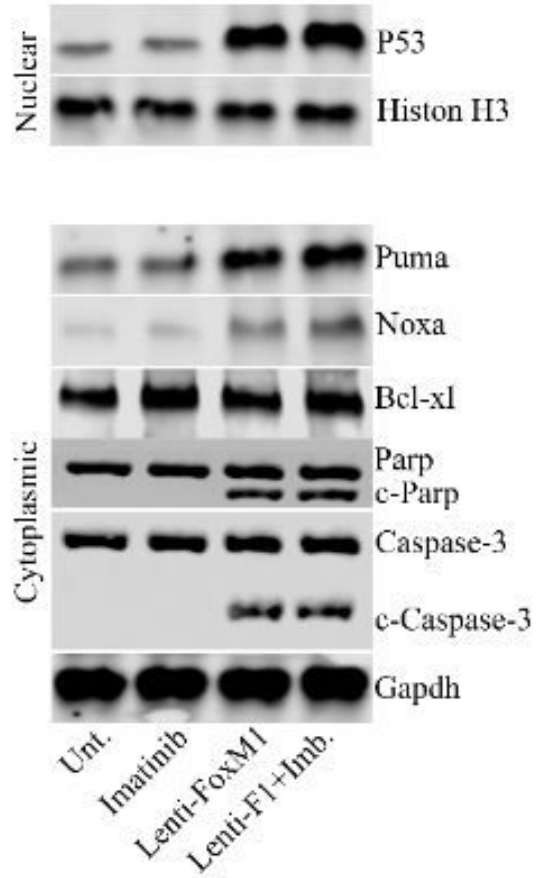

(C)

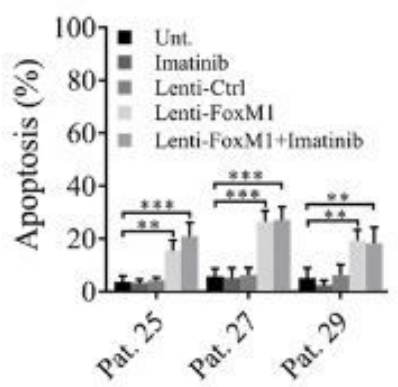

(D)

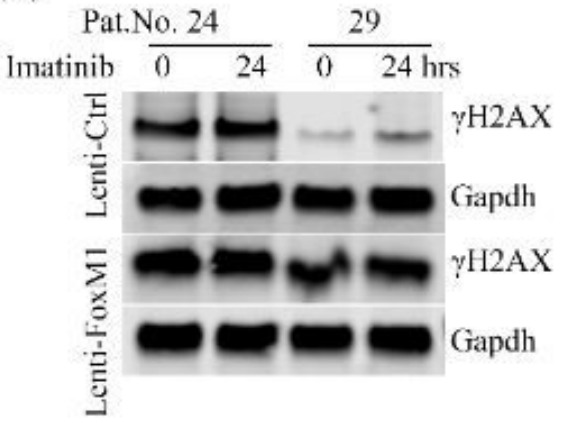

(E)

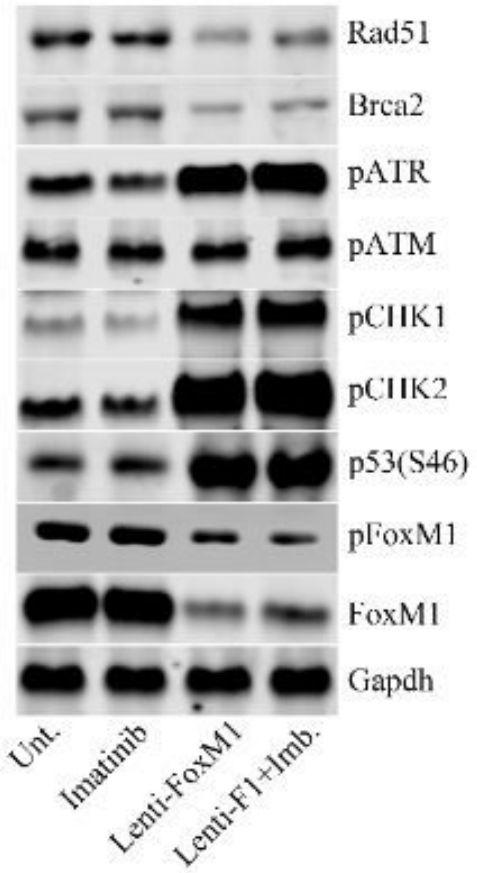

(G)

\section{$\mathrm{Bcr} / \mathrm{Abl}$}

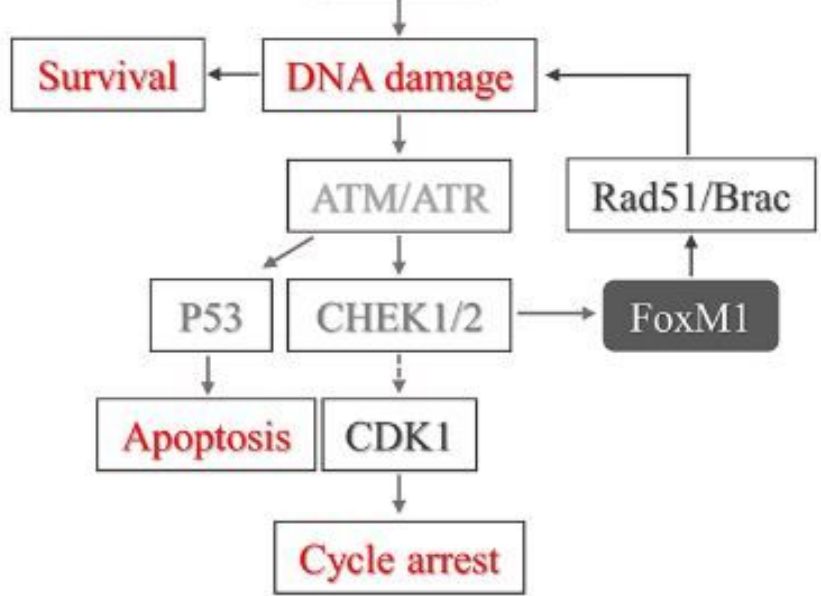

\section{Figure 5}

Repression of FoxM1 induced apoptosis in imatinib-insensitive CML cells. (A). FoxM1 phosphorylation did not affect by imatinib treatment (1 $\mu \mathrm{M}, 24$ hours) in 6 PB-MNC samples from CML patients. FDI-6 (20 $\mu \mathrm{M}, 24$ hours), a FoxM1 inhibitor, or shRNA against FoxM1 (48 hours) induced apoptosis in samples abovementioned in spite of imatinib resistance $(B, C)$. (D). FoxM1 depletion increased $\mathrm{y}-\mathrm{H} 2 \mathrm{AX}$ levels in samples. (E). FoxM1 knockdown (48 hours) suppressed the expression of Rad51 and Brca2, but caused 
more robust DDR, and lethal P53 phosphorylation on Ser 46 (patient no. 25). (F). P53 nuclear translocation and $\mathrm{Bcl}-2 /$ Caspase axis activation post FoxM1 depletion (48 hours). (G). Schematic diagram of the cytoprotective role of FoxM1 under oncogene-induced DNA damage stress. ${ }^{*} \mathrm{P}<0.05$, ${ }^{*} * \mathrm{P}<0.001$, n.s, no significant.

\section{Supplementary Files}

This is a list of supplementary files associated with this preprint. Click to download.

- Suppl.materials..pdf 